\title{
In vivo and in vitro genetic evidence of involvement of neuregulin 1 in immune system dysregulation
}

\author{
Ketan Marballi, \\ Department of Cellular and Structural Biology and Psychiatry, University of Texas Health \\ Sciences Center at San Antonio, 7703 Floyd Curl Drive, San Antonio, TX 78229, USA
}

\section{Marlon P. Quinones,}

Department of Psychiatry, University of Texas Health Sciences Center at San Antonio, 7703

Floyd Curl Drive, San Antonio, TX 78229, USA, walss@uthscsa.edu

Fabio Jimenez,

Department of Medicine, University of Texas Health Sciences Center at San Antonio, San

Antonio, TX 78229, USA

\section{Michael A. Escamilla,}

Texas Tech University Health Sciences Center, Paul L. Foster School of Medicine, Center of Excellence for Neuroscience, EI Paso, TX, USA

\section{Henriette Raventós,}

Cellular and Molecular Biology Research Center CIBCM, University of Costa Rica, Ciudad de la Investigación, San José, Costa Rica

\section{Maria Clara Soto-Bernardini, Cellular and Molecular Biology Research Center CIBCM, University of Costa Rica, Ciudad de la Investigación, San José, Costa Rica}

Seema S. Ahuja, and Department of Medicine, University of Texas Health Sciences Center at San Antonio, San Antonio, TX 78229, USA

\section{Consuelo Walss-Bass}

Department of Psychiatry, University of Texas Health Sciences Center at San Antonio, 7703 Floyd Curl Drive, San Antonio, TX 78229, USA, walss@uthscsa.edu

\section{Abstract}

Neuregulin 1 (NRG1) has been implicated in several disorders including breast cancer, multiple sclerosis, and schizophrenia. Also, recent evidence suggests that NRG1 may play a role in regulation of inflammation and immune system response. We therefore hypothesized that a schizophrenia-associated missense mutation (valine to leucine) we identified within the transmembrane region of NRG1 would also be linked to immune dysregulation. We used plasma samples from families carrying the mutation to measure levels of antibodies to 41 autoimmune markers and six cytokines (IL-1b, IL-6, IL-10, IL-8, IL-12p70, and TNF- $\alpha$ ) and used these levels 
as quantitative traits to evaluate association with the $N R G 1$ mutation, using FBAT. Next, we used Epstein-Barr virus-transformed B cells from heterozygous mutation carriers and wild-type individuals to evaluate protein and mRNA cytokine expression in vitro using quantitative PCR and ELISA assays. In vivo, increased levels of 25 autoimmune markers as well as elevated levels of cytokines were significantly associated with the $N R G 1$ mutation. In vitro, we observed a significant increase in protein secretion levels of IL- 6 , TNF- $\alpha$, and IL-8 in mutation carriers compared with controls. At the mRNA level, we observed a significant increase in IL-6 expression, while IL-4 levels appeared to be downregulated in heterozygous individuals compared with wild-type controls. This is the first report of association of a NRGI mutation with immune dysregulation. This study could contribute towards understanding the role of NRG1 in the pathogenesis of schizophrenia and other disorders in which inflammation plays an important role.

\section{Keywords}

Neuregulin-1; Immune system; Inflammation; Cytokines; Autoantibodies; Lymphoblastoid cell lines

\section{Introduction}

Neuregulin 1 (NRG1) mediates cell-cell interactions via activation of ErbB receptors, and is known to be involved in several biological processes, including cell proliferation, migration, and apoptosis, affecting multiple systems [1]. In the brain, NRG1 plays roles in processes such as neuronal migration, gliogenesis, neurotransmitter expression, and myelination, which are thought to account for its involvement in brain disorders such as multiple sclerosis and schizophrenia. More recently, several lines of evidence have emerged, suggesting that NRG1 may also be implicated in regulation of the immune response [2,4] possibly by functioning as an anti-inflammatory or antioxidant agent. Studies in rodents have shown that NRG1 has a neuroprotective role during conditions of ischemia or trauma [3]. It has been reported that human genetic variation in NRG1 modulates the effects of stress on early atherosclerosis [4], a well-established inflammatory process. Also, NRG1 has been shown to influence cell adhesion of immune cells [5] and to attenuate the release of free radicals from microglial cells [2]. More recently, it was shown that in immunedeficient mice, the levels of a splice variant of NRG1 were decreased in medial prefrontal cortices, signifying a crosstalk between NRG1 and the immune system [6].

Immune system abnormalities have been observed in patients with psychiatric disorders such as schizophrenia [7,8], as evidenced by increased expression of proinflammatory cytokines and high levels of autoantibodies. Incidence of suffering from an autoimmune disease is correlated with a $45 \%$ increase in risk for schizophrenia [9]. The mechanism by which the immune system may contribute to development of psychiatric disorders is not well understood.

We previously identified a novel NRG1 mutation associated with schizophrenia in the founder population of the Central Valley of Costa Rica (CVCR). This mutation causes the change of a residue (valine to leucine, $G>T$ ) in the transmembrane domain of the NRG1 protein [10]. The transmembrane domain is thought to be important for cleavage of NRG1 via metalloproteases and secretases. Recently, it was shown that the $V>L$ mutation affects gamma-secretase cleavage of the intracellular domain of NRG1 [11], which has been shown to act as a transcription modulator [12]. This suggests the relevance of the $V>L$ mutation in NRG1 protein function. 
Given the involvement of NRG1 in immune system response and the apparent importance of the $V>L$ mutation in NRG1 cleavage, we hypothesized that this mutation could contribute to immune system dysregulation, thereby possibly playing a role in development of immunerelated disorders, such as schizophrenia.

\section{Materials and methods}

\section{Ethics statement}

Peripheral leucocytes, DNA, and plasma were isolated from blood of subjects from the CVCR, at the time of recruitment, as previously described [10], in accordance with the principles of the Declaration of Helsinki with approval from the Institutional Review Boards of the University of Costa Rica and the University of Texas Health Science Center at San Antonio.

\section{Plasma isolation and generation of lymphoblastoid cell lines}

For plasma isolation, blood was spun within $30 \mathrm{~min}$ of collection at $3,000 \times \mathrm{g}$ for $3 \mathrm{~min}$. Plasma was aspirated by pipette and frozen in $0.5-\mathrm{mL}$ aliquots at $-80^{\circ} \mathrm{C}$ until tested. Plasma was obtained from 45 individuals, from 14 separate families (Fig. 1), for measurement of autoantibodies and cytokine levels, to be used for quantitative trait analyses, as described below.

Lymphoblastoid cell lines (LCLs) were generated from leucocytes using LeucoPREP brand cell separation tubes (Becton Dickinson Labware) and transformed using Epstein-Barr virus (EBV). For all experiments, cells were grown in RPMI 1640 medium with $2 \mathrm{mM}_{\mathrm{L}-}$ glutamine and $15 \%$ bovine growth serum at $37^{\circ} \mathrm{C}$ in a humidified $5 \% \mathrm{CO}_{2}$ chamber to a density of approximately $2 \times 10^{6}$ cells $/ \mathrm{ml}$.

Cell lines of unaffected unrelated individuals were selected for the experiments described below. All individuals had one first degree relative with psychosis. The cell lines used were from wild type ( $\mathrm{Val} / \mathrm{Val}, N=6$; three males, three females, average age at blood draw $40 \pm 20.8$ year) and heterozygous $T$ allele carriers ( $\mathrm{Val} / \mathrm{Leu}, N=5$; two males, three females, average age at blood draw $44 \pm 17$ year).

All experiments described below were repeated at least twice. While conducting the experiments, the investigators were blinded to the experimental groups.

\section{Autoimmune biomarker analysis}

A commercial multiplex assay that concurrently estimates the levels of antibodies to 43 autoimmune biomarkers (Table 1) was used (Rules-Based Medicine Inc., Austin, TX, USA). This multiplex microbead assay measures proteins in a similar manner to standard sandwich ELISA, with comparable sensitivity and range [13]. Fifty microliters of plasma was used for analysis.

\section{Fluorescence-activated cell sorting}

For cytokine quantitation, a validated cytometric bead array system (BD Biosciences) [14] was used to simultaneously measure levels of six different human cytokines from plasma samples and LCL supernatants using fluorescently conjugated detection antibodies. The minimum limits of detection (pg/ml) are 3.6, 7.2, 2.5, 3.3, 3.7, and 1.9 for IL-8, IL-1b, IL-6, IL-10, TNF- $\alpha$, and IL-12p70, respectively. Data were analyzed on a FACSCalibur flow cytometer using the BD CBA software. For IL-6, fluorescence-activated cell sorting (FACS) data were validated using the Human IL-6 ELISA Kit II (BD Biosciences). 


\section{RNA isolation and gene expression studies}

RNA isolation from LCLs was carried out using the Trizol method (Invitrogen), and samples were cleaned up using the Qiagen RNeasy plus Kit. Quantitative PCR was carried out using the RT ${ }^{2}$ Profiler PCR Array system (SABiosciences), using a 7900HT Standard Real Time PCR system (Applied Biosystems). For the pathway-based approach, we used the "Common Cytokine" array (PAHS-021, SABiosciences). Each array tests 83 different genes of interest (GOI) and five endogenous housekeeping genes (HKG) in a 96-well format. Significant array data were validated using PCR assays (SABiosciences) specific for individual cytokine genes $(I L-6, I L-4)$, with beta-2-microglobulin $(B 2 M)$ used as the endogenous HKG. Expression data were analyzed using the $\Delta \Delta \mathrm{Ct}$ method.

\section{Statistical analysis}

The autoantibody and cytokine plasma levels were used as quantitative traits to test for association with the NRG1 mutation in 14 informative CVCR families using FBAT. The program computes bi-allelic tests of association with quantitative traits, using the following commands: trait (Biomarker); offset 0.000; model additive; test bi-allelic; minsize 10; min_freq $0.000 ; P$ 1.000. Quantitative FBAT builds on the traditional transmission disequilibrium test, which compares the transmission of marker alleles from parents with affected offspring. With quantitative traits (i.e., levels of cytokines in plasma), the allelic transmissions among offspring who have high quantitative trait values are compared with transmission among offspring with low trait values $[15,16]$. In other words, the quantitative trait takes the place of affected status as the phenotype of interest.

For in vitro analyses, two-tailed, unpaired Student $t$ tests were used as appropriate. All results are expressed as mean \pm SD.

\section{Results}

\section{Autoantibody and cytokine measurements in plasma from Costa Rican families}

We first measured levels of 41 different autoantibodies (Table 1) in plasma from 14 CVCR families, informative for the $V>L$ mutation. These families were recruited and genotyped for the NRG1 mutation as previously described [10]. Of the 14 families, seven were part of the original study [10], and seven were new additional families, recruited at a later date. These 14 families were chosen, out of 206 available CVCR families, because they carried the $N R G 1$ mutation and the transmission of the rare $T$ allele from parent to offspring could be inferred, and we had plasma samples available for all genotyped family members.

The families were comprised of 45 individuals; 14 homozygous normal, 29 heterozygous, and two homozygous rare (Fig. 1). Of the 45 individuals, 14 were affected and 31 were unaffected (Fig. 1). The DSM-IV diagnoses for affected individuals in these families were as follows: schizophrenia (nine), bipolar disorder type I with psychosis (one), schizoaffective disorder depressed type (one), major depressive episode with psychosis (one), and psychotic disorder not otherwise specified (two). The $T$ allele was not found to be significantly associated with schizophrenia or psychosis in these 14 families by FBAT.

Levels of antibodies were measured in plasma from the 45 individuals by using a validated multiplex immunoassay developed by Rules-Based Medicine (www.rulesbasedmedicine.com). The mean values of antibody levels for wild type and mutants are listed in supplemental Table 1. For quantitative analysis, antibody levels were normalized using natural log. We used the normalized levels as quantitative traits to perform a quantitative family based association test (qFBAT) to evaluate association of the $N R G 1$ mutation with antibody levels. Quantitative FBAT utilizes the levels of biomarkers as the 
phenotype of interest, and therefore, analysis is done without regard to affected status. This is of importance because the goal of this study is to determine the association of the NRG1 mutation with immune dysregulation, independent of psychiatric diagnosis. Higher than expected levels of antibodies to 25 different autoimmune markers were significantly associated with the $N R G 1$ mutation ( $T$ allele; $Z$ value $>2, P<0.05$ ) in the 14 families (Table 1). Although the individual $P$ values are not corrected for multiple testing, the fact that greater than half of the markers, 25 out of 41 , showed positive association with the mutation suggested a "signature" of abnormal immune regulation in these families, leading to a generalized immune activation as manifested by increased development of antibodies against endogenous antigens. There is a possibility that the observed increased antibody levels could be due to high schizophrenia loading in these families. However, an inspection of the family pedigrees reveals that there is an equal loading of psychiatric diagnosis on $G G$ and $G T$ individuals in these families (Fig. 1).

Next, we surmised that if indeed there was a generalized immune dysregulation in carriers of the $V>L$ mutation, levels of circulating immune mediators (cytokines) should also be elevated in the 14 families. We measured levels of six different cytokines (IL-1b, IL-6, IL-10, IL-8, IL-12p70, and TNF- $\alpha$ ) in plasma from the 45 individuals, using a cytometric bead array system followed by FACS analysis. The mean cytokine levels for each genotype group are listed in supplemental Table 1. Statistical analysis using qFBAT indicated that higher than expected levels of IL-1b, IL-6, IL-10, IL-12p70, and TNF- $\alpha$ were significantly associated $(Z$ value $>2, P<0.05$ ) with the $N R G 1$ mutation in these 14 families (Table 1 ).

\section{Immunological profiling of lymphoblastoid cell lines}

Considering that multiple confounding factors could affect measurements of antibody titers and cytokines in vivo, we sought to obtain more direct evidence for an effect of NRG1 in vitro by comparing the immunological features of EBV-transformed $\mathrm{B}$ cells derived from heterozygous vs. wild-type individuals. The study of LCLs is uniquely suited to the investigation of genetic polymorphisms in immune activation. In vitro, the EB virus infects resting (naive or memory) B lymphocytes and transforms them into immortalized, polyclonal LCLs. Regardless of their origin, most EB virus genome-carrying cell lines are thought to contain B cells that are "frozen" at the lymphoblast stage of differentiation [17]. In other words, the analysis of the immune phenotype of LCLs can provide information about the overall immune activation of the donor at the time of sample collection. Furthermore, LCLs are ideal for the study of the effects of polymorphisms in the transcriptome. Concurrent genome-wide SNP and expression studies revealed that genes expressed in LCLs are enriched in gene ontology categories of immune response, and there is a significant heritability for these traits, emphasizing the value of the use of LCLs for the study of genetic variation in immunity [18]. In addition, data mining mRNA expression arrays of LCLs from $15 \mathrm{CEPH/Utah}$ families revealed expression of NRG1 [18], suggesting that LCLs could be informative about the effects of the NRGIV>L mutation in immune dysregulation. [Gene Expression Omnibus database, DataSet GDS1048, (http://www.ncbi.nlm.nih.gov/geo.)].

\section{Measurement of cytokine expression levels in LCLs}

We first measured expression of IL-8, IL-1b, IL-6, IL-10, TNF- $\alpha$, and IL-12p70 in supernatants from LCLs of wild-type $(\mathrm{Val} / \mathrm{Val}, \mathrm{N}=6)$ and heterozygous $T$ allele carriers ( Val/ Leu, $\mathrm{N}=5$ ). The cell lines were chosen from unrelated, unaffected individuals, each with a first degree relative with psychosis. Because the family loading for psychosis is equal for all samples and the individuals themselves do not suffer from any psychiatric disorder, we can directly investigate the effect of the $V>L$ mutation on cytokine expression, without the confounding factor of an existing psychiatric disorder. The five $T$ allele carriers were 
chosen from the 14 informative families described above, each from a different family. As described, only two homozygous mutation carriers have been identified in the CVCR population. Both of these individuals have a schizophrenia diagnosis. Given that the present experimental design excluded affected individuals, for all experiments described below, only heterozygous carriers were used.

Equal numbers of cells were plated overnight, and cytokine levels were evaluated from supernatants, $24 \mathrm{~h}$ after plating, using FACS. Cell counts were not found to be different between wild type and mutants $24 \mathrm{~h}$ after plating (data not shown). We found that, compared with LCLs from wild-type carriers, LCLs from $\mathrm{T}$ allele carriers produced significantly higher levels of proinflammatory cytokines (Fig. 2a). TNF- $\alpha$, IL-6, and IL-8 showed significantly higher levels in the mutants compared with controls (two-tailed Student $t$, $P=0.0187,0.0166,0.0323$, respectively).

We validated the IL-6 results using an IL-6-specific ELISA. IL-6 supernatant levels, normalized to total protein content, were observed to be significantly higher in mutants compared with controls, confirming the results obtained in the six-cytokine kit (two-tailed Student $t, P=0.008$ ) (Fig. 2b).

Next, a cytokine gene array (SABiosciences) was used to evaluate 83 cytokine mRNA levels by quantitative PCR in the same cell lines as above, wild-type $(N=6)$ and $T$ allele carriers $(N=5)$. Four genes, $I L-4, I L-6, I F N A 2$, and $B M P 8 B$, showed a significant difference between the mutants and controls (Table 2).

The $I L-4$ and $I L-6$ results were validated using specific single gene assays. cDNA generated from each individual cell line was assayed in duplicate using $B 2 M$ as the endogenous housekeeping control. We observed a 4.39-fold down-regulation of $I L-4$ and a 35.46-fold up-regulation of $I L-6$ in mutants compared with controls (two-tailed Student $t, P=0.014$ and 0.0011 for $I L 4$ and $I L-6$, respectively) (Table 3 ).

\section{Discussion}

Because of its multiple isoforms and various functional roles, NRG1 has been implicated in the pathophysiology of a wide variety of disorders, including breast cancer [19] schizophrenia and bipolar disorder [20] in human studies, and atherosclerosis [21], myocardial dysfunction [22] and multiple sclerosis [23] in animal models. Interestingly, immune system dysregulation has been implicated as a risk factor in all of these disorders. Inflammation has been associated with poor prognosis in breast cancer [24].

Proinflammatory imbalance and uncontrolled immune response have been postulated as the cause of acute myocardial infarction [25]. In schizophrenia, immune system abnormalities such as increased expression of proinflammatory cytokines and high levels of autoantibodies have been repeatedly reported [7,8]. Multiple sclerosis is characterized by immune systemmediated demyelination and axonal loss. In an animal model of multiple sclerosis, Marchionni et al. demonstrated that treatment with recombinant NRG1 caused greater remyelination and decreased relapse rate, compared with untreated controls [23]. Increased expressions of myelin basic protein and IL-10 were also observed. The modulation of myelination by NRG1 in the CNS (but not in the PNS) is thought to be independent of NRG1 ErbB signaling [26].

Our present results suggest the possibility that the NRG1 $V>L$ mutation may be involved in causing immune system dysregulation, which then, in combination with different specific sets of genes and environmental factors, may lead to development of a particular disorder. The NRGI $V>L$ mutation was first identified in a genetic study searching for schizophreniacausative mutations [10]. Although the $N R G 1$ gene is one of the most promising 
schizophrenia candidate genes identified to date, no definite causative mutations within this gene have been identified. Therefore, research aimed at determining the functional role of $N R G 1$ polymorphisms is an area of much interest. A deeper understanding of the biological functionality of $N R G 1$ variants could help to understand the role that NRG1 may play in different pathological conditions.

In this study, we aimed to determine whether the $V>L$ polymorphism could be associated with immune system alterations. Given that this mutation occurs in the transmembrane region of NRG1 and knocking out this region has been shown to cause schizophrenia-like behavior in mice [27], we focused exclusively on this transmembrane mutation. However, it is highly possible that other $N R G 1$ variants, as well as SNPs within other schizophrenia candidate genes (i.e., DISC1, dysbindin, and COMT), may also be associated with immune system dysregulation. To our knowledge, SNPs in schizophrenia candidate genes other than cytokines have not been previously reported to be associated with alterations in the immune system. We hope our present results will encourage other researchers to investigate this possibility.

We observed statistical evidence of increased plasma autoantibodies and proinflammatory cytokines, including IL-6, TNF- $\alpha$, and IL- $1 \mathrm{~b}$ in carriers of the $V>L$ mutation.

Polymorphisms within $I L-6$ and $I L-1 b$ have been associated with schizophrenia [28,29]. Interestingly, it has been shown that polymorphisms in $I L-I b$ and $N R G I$ genes interact statistically with each other and are linked to schizophrenia [29]. Increased levels of proinflammatory cytokines, in particular IL- 6 and TNF- $\alpha$, have been repeatedly observed in schizophrenia patients [8]. IL-6 has also been implicated in worsening of autoimmune disorders by causing blood-brain barrier disturbances and increased IgG secretion [8]. In a large linkage study of Danish National Registers, a 45\% greater risk of developing schizophrenia in families with a history of autoimmune disorders was reported [9]. Furthermore, high levels of autoantibodies, including anticardiolipin, anti-histone, antiDNA, and anti-nuclear antibodies, have been reported in schizophrenic patients. Serum antibodies against brainspecific regions such as the hippocampus, amygdala, and frontal cortex have also been reported [8]. Na and Kim [30] reported reduced levels of IL-4, an antiinflammatory cytokine, in serum of schizophrenia patients compared with controls. This correlates with our present data showing reduced $I L-4$ expression in cell lines of carriers of the $V>L$ mutation, compared with wild-type carriers (Fig. 2). Altogether, these studies support the hypothesis that an overactive immune system could be an underlying causative factor in schizophrenia development. Our current findings suggest that this over-activity could be in part due to the $N R G I V>L$ mutation. Further studies with additional and larger sets of samples from different populations need to be performed in order to corroborate these results.

Investigation of the specific mechanism by which the $V>L$ mutation leads to increased immune activation is currently underway in our laboratory. One possibility is that the change of $V>L$ alters the proteolysis of NRG1 transmembrane proteins, resulting in different availability of bioactive extracellular and intracellular domains of NRG1, which could possibly lead to immune dysregulation. The NRG1 transmembrane domain interacts with metalloproteinases, such as $\beta$ and $\gamma$ secretase, which form the bioactive extracellular and intracellular domains of the proteins. The cleaved extracellular NRG1 domain interacts with ErbB receptors and activates numerous downstream pathways, such as the AKT and ERK pathways, which are involved in regulation of gene expression [31], and in leukocyte activation and survival. In several different strains of mice that exhibit lupus-like phenotypes, the level of phosphorylation of AKT and ERK in unstimulated B cells was significantly elevated [32]. Moreover, the ERK pathway has been shown to regulate cytokine expression [33]. 
The intracellular NRG1 domain, formed via $\gamma$ secretasemediated cleavage, migrates to the nucleus and acts as a transcription factor, regulating mRNA expression of genes such as $B C L-X_{L}, B A K, R I P$, and $O C T-3$ [12]. Importantly, a recent study showed that the NRG1 $V>L$ mutation reduces $\gamma$-secretase cleavage in COS-1 cells [11], suggesting that this mutation alters the function of the NRG1 protein. Differential availability of intracellular NRG1 associated with the $V>L$ mutation may result in dysregulation of the mechanisms underlying elimination of self-reactive B cells, which are mostly dependent on the counterbalancing effects of pro-apoptotic proteins such BAK and anti-apoptotic proteins such as BCL-XL [34]. Persistence of self-reactive B cells may lead to autoantibody production and in certain cases full-blown autoimmune disorders [34].

Given that both the extracellular and intracellular NRG1 pathways are involved in regulation of gene expression, it is possible that cytokine expression is regulated by either, or both, of these pathways. Interestingly, IL-6 and IFN- $\gamma$ have recently been shown to induce NRG1 cleavage in endothelial cells and enhance angiogenesis via NRG1 signaling in vitro [35]. Therefore, it is possible that the increase in IL-6 expression we have observed in the present study may be a feedback mechanism compensating for reduced NRG1 cleavage due to the $V>L$ mutation. Further experiments need to be carried out to determine the exact mechanism by which NRG1 may regulate cytokine expression.

Given the small sample sizes used in this study, our present results must be interpreted with caution and should be replicated by independent samples. In addition, studies investigating the potential association of other NRG1 polymorphisms with immune system dysregulation are warranted.

In conclusion, our study is the first to report statistical association between a mutation in NRG1 and immune system dysregulation. These findings could aid in the understanding of the molecular mechanisms of inflammatory regulated disorders in which NRG1 plays an important role.

\section{Supplementary Material}

Refer to Web version on PubMed Central for supplementary material.

\section{Acknowledgments}

This work was supported by grants from the National Institutes of Health (K01MH077777), the Stanley Medical Research Institute, and NARSAD, the mental health research association to CWB. We thank the personnel of the CIBCM at the University of Costa Rica for their assistance in collecting the samples.

\section{References}

1. Falls DL. Neuregulins: functions, forms, and signaling strategies. Exp Cell Res. 2003; 284:14-30. [PubMed: 12648463]

2. Dimayuga FO, Ding Q, Keller JN, Marchionni MA, Seroogy KB, Bruce-Keller AJ. The neuregulin GGF2 attenuates free radical release from activated microglial cells. J Neuroimmunol. 2003; 136:67-74. [PubMed: 12620644]

3. Li Y, Xu Z, Ford GD, Croslan DR, Cairobe T, Li Z, Ford BD. Neuroprotection by neuregulin-1 in a rat model of permanent focal cerebral ischemia. Brain Res. 2007; 1184:277-283. [PubMed: 17961519]

4. Hintsanen M, Elovainio M, Puttonen S, Kivimaki M, Raitakari OT, Lehtimaki T, Rontu R, Juonala M, Kahonen M, Viikari J, et al. Neuregulin-1 genotype moderates the association between job strain and early atherosclerosis in young men. Ann Behav Med. 2007; 33:148-155. [PubMed: 17447867] 
5. Kanakry CG, Li Z, Nakai Y, Sei Y, Weinberger DR. Neuregulin-1 regulates cell adhesion via an ErbB2/phosphoinositide-3 kinase/Akt-dependent pathway: potential implications for schizophrenia and cancer. PLoS ONE. 2007; 2:e1369. [PubMed: 18159252]

6. Asp L, Beraki S, Kristensson K, Ogren SO, Karlsson H. Neonatal infection with neurotropic influenza A virus affects working memory and expression of type III Nrg1 in adult mice. Brain Behav Immun. 2009; 23:733-741. [PubMed: 19362585]

7. Potvin S, Stip E, Sepehry AA, Gendron A, Bah R, Kouassi E. Inflammatory cytokine alterations in schizophrenia: a systematic quantitative review. Biol Psychiatry. 2008; 63:801-808. [PubMed: 18005941]

8. Strous RD, Shoenfeld Y. Schizophrenia, autoimmunity and immune system dysregulation: a comprehensive model updated and revisited. J Autoimmun. 2006; 27:71-80. [PubMed: 16997531]

9. Eaton WW, Byrne M, Ewald H, Mors O, Chen CY, Agerbo E, Mortensen PB. Association of schizophrenia and autoimmune diseases: linkage of Danish national registers. Am J Psychiatry. 2006; 163:521-528. [PubMed: 16513876]

10. Walss-Bass C, Liu W, Lew DF, Villegas R, Montero P, Dassori A, Leach RJ, Almasy L, Escamilla $\mathrm{M}$, Raventos $\mathrm{H}$. A novel missense mutation in the transmembrane domain of neuregulin 1 is associated with schizophrenia. Biol Psychiatry. 2006; 60:548-553. [PubMed: 16730337]

11. Dejaegere T, Serneels L, Schafer MK, Van Biervliet J, Horre K, Depboylu C, Alvarez-Fischer D, Herreman A, Willem M, Haass C, et al. Deficiency of Aph1B/C-gamma-secretase disturbs Nrg1 cleavage and sensorimotor gating that can be reversed with antipsychotic treatment. Proc Natl Acad Sci USA. 2008; 105:9775-9780. [PubMed: 18626010]

12. Bao J, Wolpowitz D, Role LW, Talmage DA. Back signaling by the Nrg-1 intracellular domain. J Cell Biol. 2003; 161:1133-1141. doi:10.1083/jcb.200212085jcb.200212085. [PubMed: 12821646]

13. Camargo JF, Quinones MP, Mummidi S, Srinivas S, Gaitan AA, Begum K, Jimenez F, Van Compernolle S, Unutmaz D, Ahuja SS, et al. CCR5 expression levels influence NFAT translocation, IL-2 production, and subsequent signaling events during T lymphocyte activation. J Immunol. 2009; 182:171-182. [PubMed: 19109148]

14. Morgan E, Varro R, Sepulveda H, Ember JA, Apgar J, Wilson J, Lowe L, Chen R, Shivraj L, Agadir A, et al. Cytometric bead array: a multiplexed assay platform with applications in various areas of biology. Clin Immunol. 2004; 110:252-266. [PubMed: 15047203]

15. Lange C, DeMeo DL, Laird NM. Power and design considerations for a general class of familybased association tests: quantitative traits. Am J Hum Genet. 2002; 71:1330-1341. [PubMed: 12454799]

16. Abecasis GR, Cardon LR, Cookson WO. A general test of association for quantitative traits in nuclear families. Am J Hum Genet. 2000; 66:279-292. [PubMed: 10631157]

17. Yu Y, Rabinowitz R, Steinitz M, Schlesinger M. Correlation between the expression of CD4 and the level of CD4 mRNA in human B-cell lines. Cell Immunol. 2002; 215:78-86. [PubMed: 12142039]

18. Monks SA, Leonardson A, Zhu H, Cundiff P, Pietrusiak P, Edwards S, Phillips JW, Sachs A, Schadt EE. Genetic inheritance of gene expression in human cell lines. Am J Hum Genet. 2004; 75:1094-1105. [PubMed: 15514893]

19. Hayes NV, Gullick WJ. The neuregulin family of genes and their multiple splice variants in breast cancer. J Mammary Gland Biol Neoplasia. 2008; 13:205-214. [PubMed: 18415007]

20. Georgieva L, Dimitrova A, Ivanov D, Nikolov I, Williams NM, Grozeva D, Zaharieva I, Toncheva D, Owen MJ, Kirov G, O'Donovan MC. Support for neuregulin 1 as a susceptibility gene for bipolar disorder and schizophrenia. Biol Psychiatry. 2008; 64:419-427. [PubMed: 18466881]

21. Clement CM, Thomas LK, Mou Y, Croslan DR, Gibbons GH, Ford BD. Neuregulin-1 attenuates neointimal formation following vascular injury and inhibits the proliferation of vascular smooth muscle cells. J Vasc Res. 2007; 44:303-312. [PubMed: 17438359]

22. Freedman NJ, Ginsburg GS. Novel- and "neu"-therapeutic possibilities for heart failure. J Am Coll Cardiol. 2006; 48:1448-1450. [PubMed: 17010809]

23. Marchionni MA, Cannella B, Hoban C, Gao YL, Garcia-Arenas R, Lawson D, Happel E, Noel F, Tofilon P, Gwynne D, Raine CS. Neuregulin in neuron/glial interactions in the central nervous system. GGF2 diminishes autoimmune demyelination, promotes oligodendrocyte progenitor 
expansion, and enhances remyelination. Adv Exp Med Biol. 1999; 468:283-295. [PubMed: 10635037]

24. Hojilla CV, Wood GA, Khokha R. Inflammation and breast cancer: metalloproteinases as common effectors of inflammation and extracellular matrix breakdown in breast cancer. Breast Cancer Res. 2008; 10:205. [PubMed: 18394187]

25. Bodi V, Sanchis J, Nunez J, Mainar L, Minana G, Benet I, Solano C, Chorro FJ, Llacer A. Uncontrolled immune response in acute myocardial infarction: unraveling the thread. Am Heart J. 2008; 156:1065-1073. [PubMed: 19033000]

26. Brinkmann BG, Agarwal A, Sereda MW, Garratt AN, Muller T, Wende H, Stassart RM, Nawaz S, Humml C, Velanac V, Radyushkin K, Goebbels S, Fischer TM, Franklin RJ, Lai C, Ehrenreich H, Birchmeier C, Schwab MH, Nave KA. Neuregulin-1/ErbB signaling serves distinct functions in myelination of the peripheral and central nervous system. Neuron. 2008; 59:581-595. doi:10.1016/ j.neuron.2008.06.028. [PubMed: 18760695]

27. O'Tuathaigh CM, O'Connor AM, O'Sullivan GJ, Lai D, Harvey R, Croke DT, Waddington JL. Disruption to social dyadic interactions but not emotional/anxiety-related behaviour in mice with heterozygous 'knockout' of the schizophrenia risk gene neuregulin-1. Prog Neuropsychopharmacol Biol Psychiatry. 2008; 32:462-466. doi:10.1016/j.pnpbp.2007.09.018. [PubMed: 17980471]

28. Paul-Samojedny M, Kowalczyk M, Suchanek R, Owczarek A, Fila-Danilow A, Szczygiel A, Kowalski J. Functional polymorphism in the interleukin-6 and interleukin-10 genes in patients with paranoid schizophrenia—a case-control study. J Mol Neurosci. 2010 doi:10.1007/ s12031-010-9365-6.

29. Hanninen K, Katila H, Saarela M, Rontu R, Mattila KM, Fan M, Hurme M, Lehtimaki T. Interleukin-1 beta gene polymorphism and its interactions with neuregulin-1 gene polymorphism are associated with schizophrenia. Eur Arch Psychiatry Clin Neurosci. 2008; 258:10-15. [PubMed: 17901998]

30. Na KS, Kim YK. Monocytic, Th1 and th2 cytokine alterations in the pathophysiology of schizophrenia. Neuropsychobiology. 2007; 56:55-63. [PubMed: 18037815]

31. Mei L, Xiong WC. Neuregulin 1 in neural development, synaptic plasticity and schizophrenia. Nat Rev Neurosci. 2008; 9:437-452. [PubMed: 18478032]

32. Wu T, Qin X, Kurepa Z, Kumar KR, Liu K, Kanta H, Zhou XJ, Satterthwaite AB, Davis LS, Mohan C. Shared signaling networks active in B cells isolated from genetically distinct mouse models of lupus. J Clin Invest. 2007; 117:2186-2196. [PubMed: 17641780]

33. Ohnishi H, Takeda K, Domenico J, Lucas JJ, Miyahara N, Swasey CH, Dakhama A, Gelfand EW. Mitogenactivated protein kinase/extracellular signal-regulated kinase 1/ 2-dependent pathways are essential for CD8+ T cell-mediated airway hyperresponsiveness and inflammation. J Allergy Clin Immunol. 2009; 123:249-257. [PubMed: 19130938]

34. Deming PB, Rathmell JC. Mitochondria, cell death, and B cell tolerance. Curr Dir Autoimmun. 2006; 9:95-119. [PubMed: 16394657]

35. Kalinowski A, Plowes NJ, Huang Q, Berdejo-Izquierdo C, Russell RR, Russell KS. Metalloproteinase-dependent cleavage of neuregulin and autocrine stimulation of vascular endothelial cells. FASEB J. 2010; 24 000-000. 

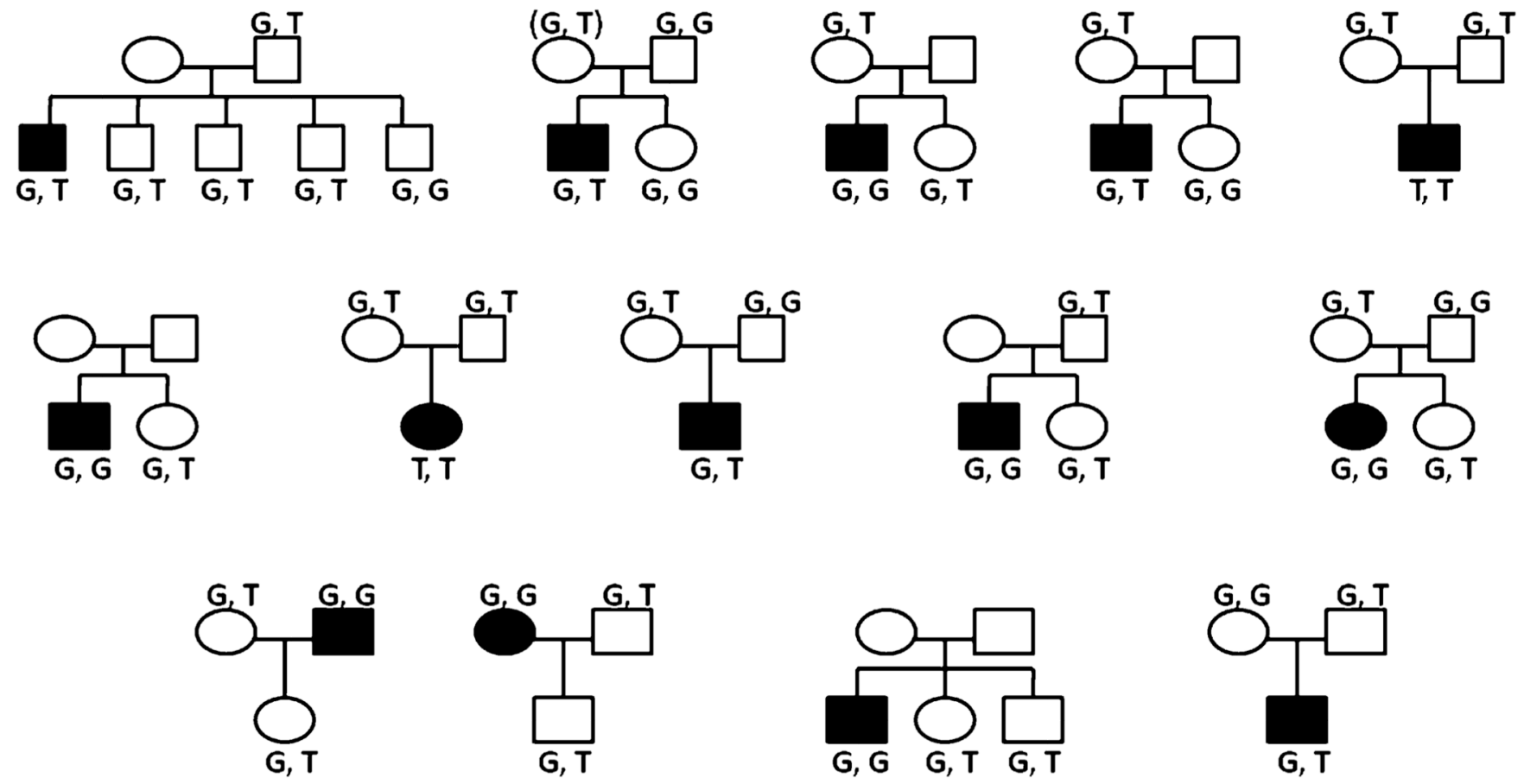

Fig. 1.

Pedigrees of informative families for the NRG1 $G>T$ polymorphism. Subjects in black were diagnosed as having a psychotic disorder $(N=14)$, and subjects in white were diagnosed as unaffected $(N=31)$. Subjects not containing allele information were not genotyped. Genotypes in parentheses were inferred. DSM-IV consensus diagnoses of the 14 affected individuals were as follows: schizophrenia, nine; schizoaffective depressed, one; bipolar disorder with psychosis, one; psychosis not otherwise specified, two; major depressive episode with psychosis, one 

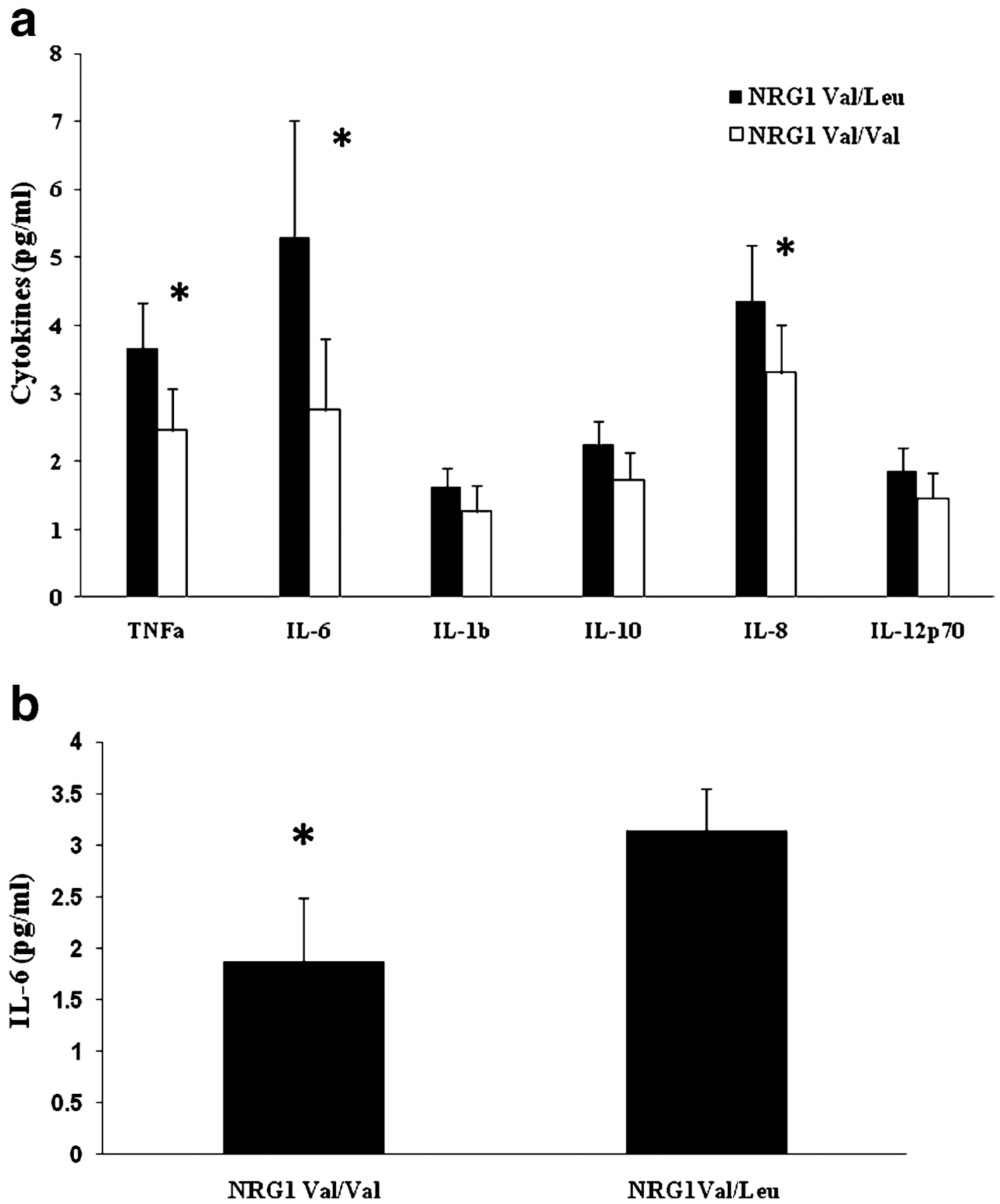

Fig. 2.

Expression of cytokines in lymphoblastoid cell lines. a Measurement of cytokine levels using FACS in cell supernatants from LCLs of carriers of the NRG1 mutation compared with controls. $P=0.0187,0.0166$, and 0.0323 , for TNF- $\alpha$, IL-6, and IL-8, respectively (twotailed Student $t$ ). b Measurement of IL-6 levels using ELISA in cell supernatants from LCLs of carriers of the NRG1 mutation and controls. $P=0.008$ (two-tailed Student $t$ ). Val/Leu, $N=5$ and $\mathrm{Val} / \mathrm{Val}, N=6$. All concentration values are expressed in $\mathrm{pg} / \mathrm{ml}$. Data represent logtransformed values. Asterisks represent significant $P$ value 


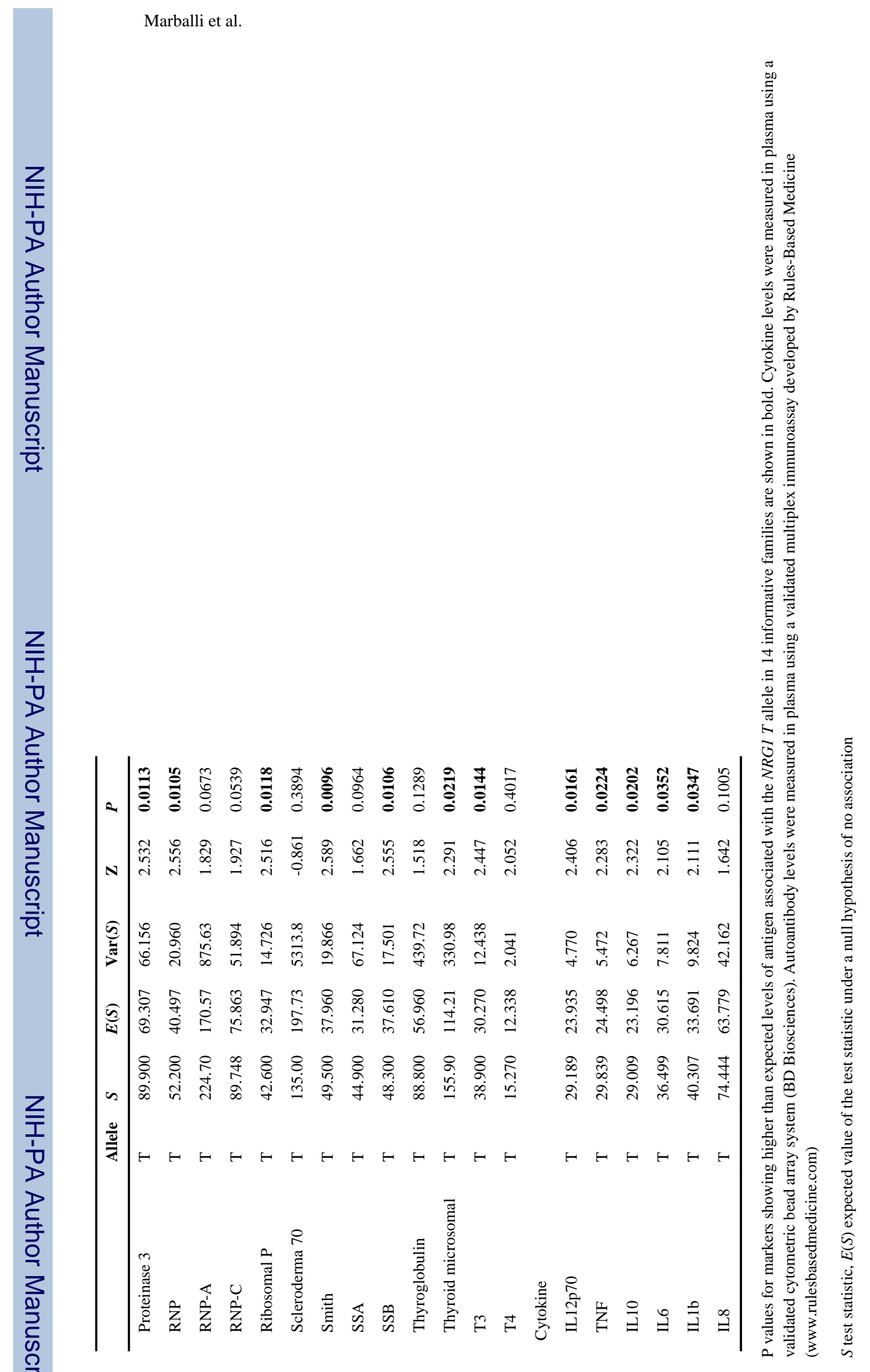

Page 14 


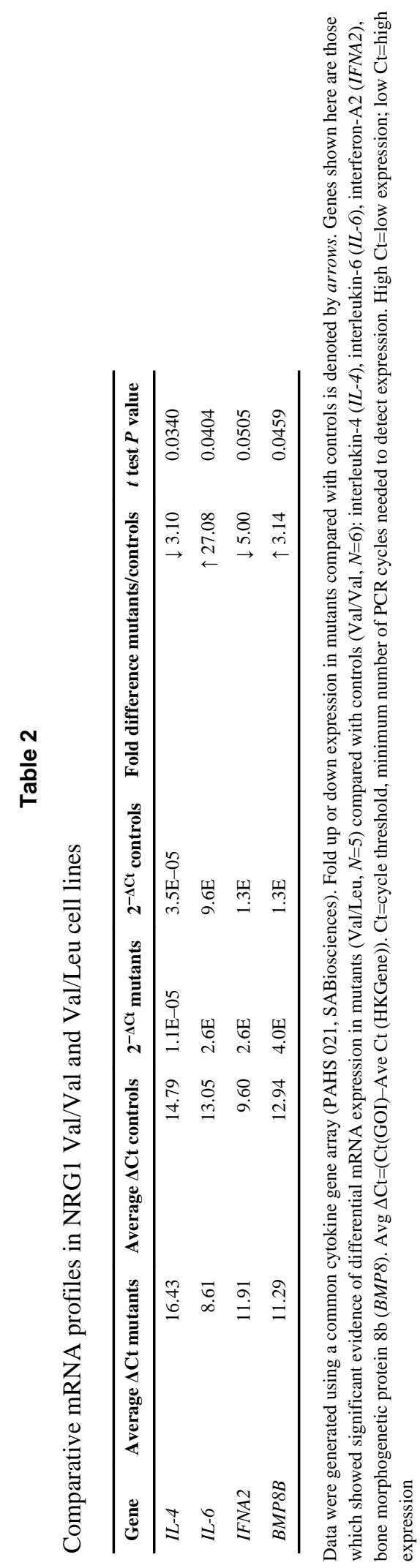

$J$ Mol Med (Berl). Author manuscript; available in PMC 2011 November 1. 


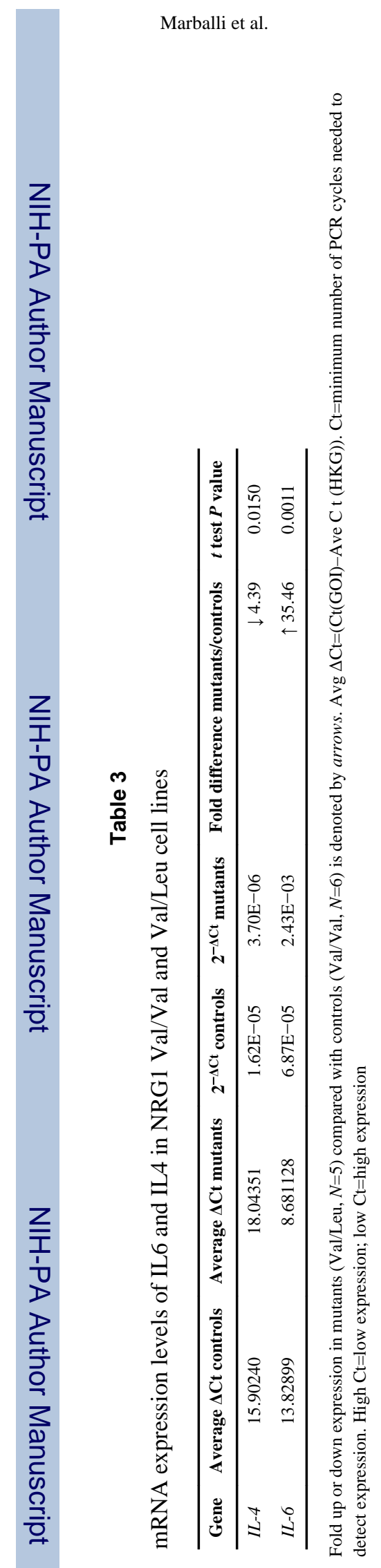

J Mol Med (Berl). Author manuscript; available in PMC 2011 November 1. 